\title{
Development of Web-based Expert System for Convenient Precise Positioning
}

\author{
Kap-Yong Jung ${ }^{1}$ and Joon-Kyu Park ${ }^{2 *}$ \\ ${ }^{1}$ Dept. of Civil Engineering, Chungnam National University \\ 99 Daehak-ro, Yuseong-gu, Daejeon, Korea \\ ${ }^{2}$ Dept. of Civil Engineering, Seoil University, \\ 28, Yongmasan-ro 90-gil, Jungnang-gu, Seoul, Korea \\ jungjusa@hanmail.net,jkpark@seoil.ac.kr
}

\begin{abstract}
GPS is a satellite-based navigation system capable of precise positioning. GPS can be applied to various areas such as setup of international terrestrial reference frame, earth axis' rotation coefficient determination, earthquake and crustal movements sensing, etc. But to gain precise results through GPS data processing, comprehensive expertise and a lot of time are necessary to determine parameters for processing and professional software usage, etc. Therefore, this research tried to develop a professional positioning system capable of easy and precise positioning for nonprofessionals. In this research, we developed a specialized precise positioning system where GPS data are fed for automatic data processing and results based on the web. Also we compared the system developed herein with other existing positioning processes to explore system application.
\end{abstract}

Keywords: Expert system, GPS, Data processing, Precise positioning

\section{Introduction}

GPS determining time and position based on satellite signals was first developed for a military purpose. After the 1980s, GPS has been utilized in diverse areas such as geodesic surveying and measurement, geophysics and weather forecast. In industrial fields, demand for GPS has surged explosively mainly in automobile, airplane and vessel navigation, ubiquitous, GIS, etc. [1]. In particular, GPS has been recognized as a prerequisite for precise positioning [2-5]. At the end of the 1980s, GPS related researches began on automobile navigation, geodesic measurement, geophysics, etc. along with data process related researches [6]. Softwares for an academic purpose and commercial softwares were analyzed according to positioning conditions to verify the limitations of commercial softwares and precise positioning application [7], and online data processing service was also researched for evaluation [8]. Existing GPS data processing related researches, however, mostly used academic or commercial softwares to process data and compare the programs. Only less than insufficient amount of researches have been conducted on developing a system capable of precise positioning and data processing even by nonprofessionals. For GPS-based precise outcome calculation, comprehensive expertise and lots of time are required such as data processing parameter determination and specialized software usage. Therefore, a new

* Corresponding Author 
automated system is necessary for nonprofessionals as well. In this understanding, this research tried to develop a specialized automated precise positioning system based on the web and compare it with other GPS data processing processes to suggest the applicability of the developed expert system herein. Figure 1 shows research flow.

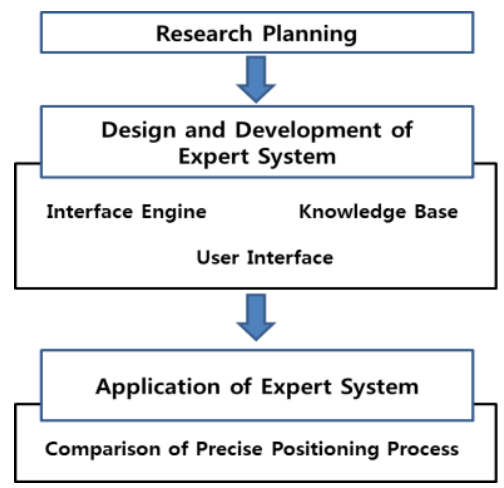

Figure 1. Research flow chart

\section{Development of Expert System}

This research developed an expert system based on the web guaranteeing an easy access to nonprofessionals. The expert system is used to resolve a problem in a specific area requiring expertise and comprises of 3 parts of inference engine, knowledge base and user interface. The inference engine refers to software containing measures for GPS data processing and we employed Bernese for precise science technology by Swiss Bern University to develop the expert system. Bernese's GPS data processing process is shown in Figure 2 [9].

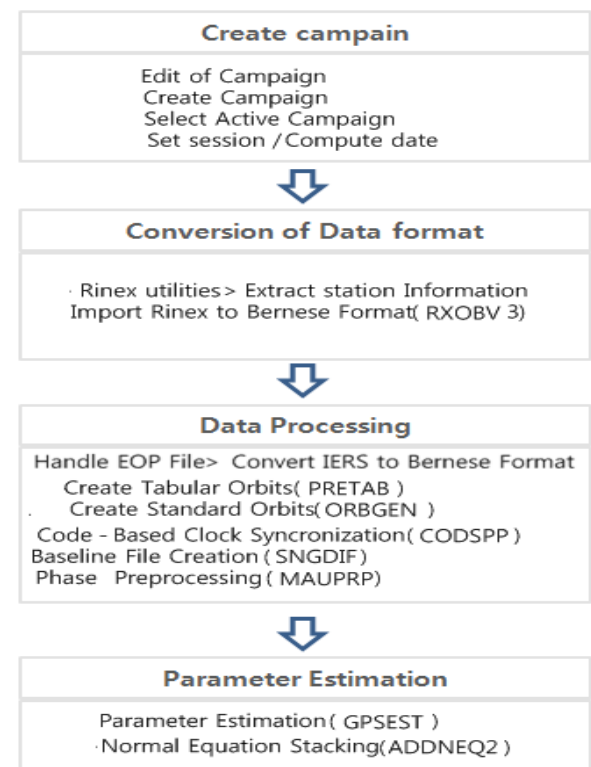

Figure 2. Data processing flow of Bernese

The knowledge base holds parameters necessary for the inference engine to resolve problems. Ephemeris, satellite clock anomalies, ionospheric model, earth rotation parameters, 
GPS code bias, etc. are the knowledge base. Each parameter is provided through IGS, Bern University, and NASA JPL. Table 1 displays parameters for precise positioning.

Table 1. Parameters for precise positioning

\begin{tabular}{cc}
\hline Parameters & Description \\
\hline Satellite Ephemeris & Precise Ephemeris \\
\hline Ambiguity Resolution & Quasi Ionosphere Free \\
\hline Tropospheric Correction & Dry and Wet Niell Model \\
\hline Earth Gravity Potential & JGM3 \\
\hline Sub-daily Earth rotation parameters & IERS2000 \\
\hline Nutation & IAU2000 \\
\hline Antenna Model & Absolute Model \\
\hline Solar System Ephemerides & JPL DE200
\end{tabular}

The user interface is a contact face between the expert system and users. Herein MCV(Model, Controller, View) was employed. The MCV model is an architecture pattern used in software engineering [10]. This pattern separates business logic from the user interface and enables application development which is easily modifiable without mutual interference between an application's visual aspects or business logics going under it. In the MVC, Model represents application data, View represents user interface factors such as text, check box items, etc. and Controller manages the mutual interaction between data and business logic. Figure 3 shows the information flow between the MCV model and user interface.

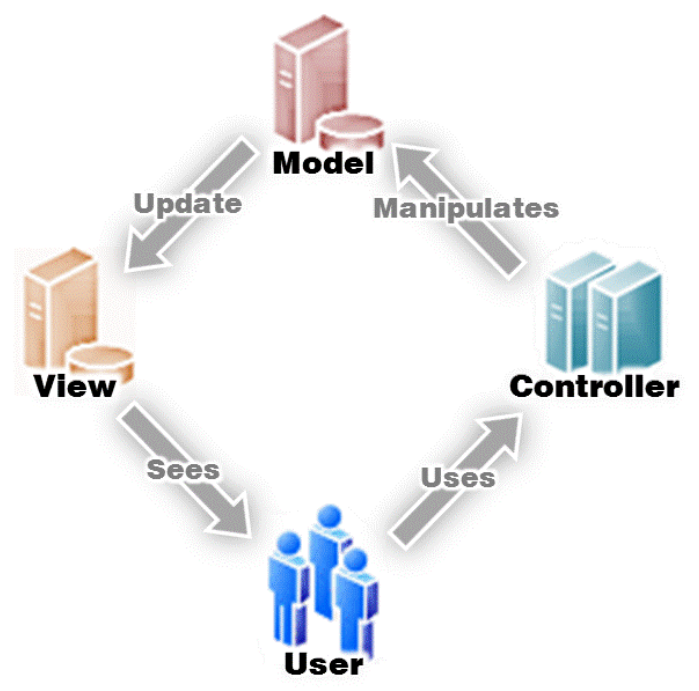

Figure 3. Information flow between MCV model and user

Model is an application's business logic or an aggregate of components for an object or data processing. Control is responsible for object or data flow. View takes care of object or data generation normally managing data input and output. Model processes and treats the object or data delivered from Controller according to application business logics and sends changed status of object to View or Controller. 
Controller is a component managing object or data flow. Controller understands the messages transferred from View and determines which model to send the corresponding object and deliver a necessary object to models. View is related to object or data generation, input and output. View can request the status of an object to a model upon necessity and take different forms of output according to the status of an answer. Figure 4 represents object and data flow between Model, Controller and View.

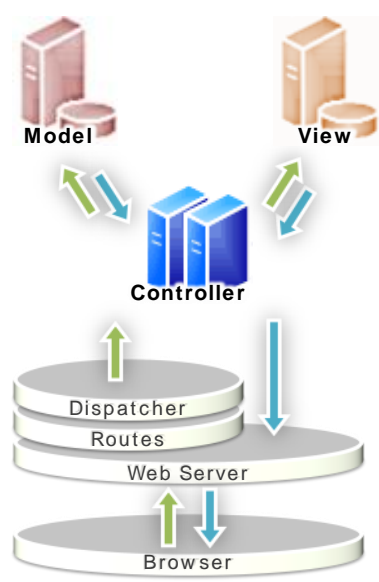

Figure 4. Objects and data flow between Model, Controller and View

The expert system's data processing is consisted of information input, data generation, data download, data processing and result output. User input items were minimized and maintenance and upgrade were designed easy. Figure 5 shows the source composition of the expert system corresponding to the MCV model.

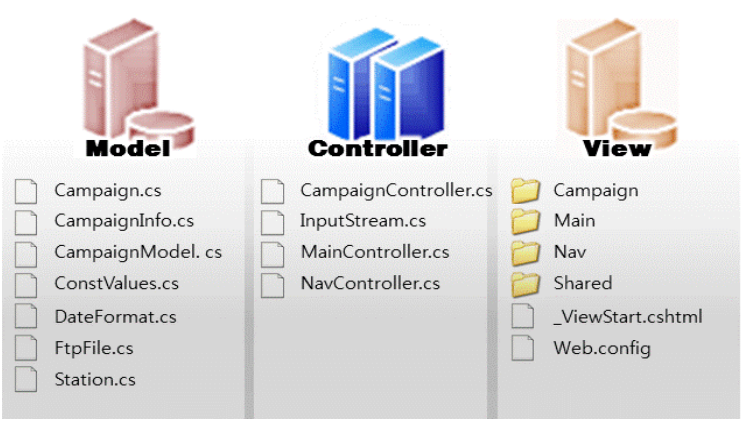

Figure 5. Source composition of the expert system about MCV model

Model contains information on campaign, campaign information, observatory, GPS data, day of year, etc. Main Controller of the Controller, deals with index page, introduction page, about page and contact page. Campaign Controller analyzes observation files. The expert system's web screen is consisted of Introduction, About GPS, Submit Data, Contact us menus. On the lower part of the screen, links to NGI(National Geographic Information Institute), IGS, Bern University, NASA JPL were set. Figure 6 shows the basic screen of the expert system. 


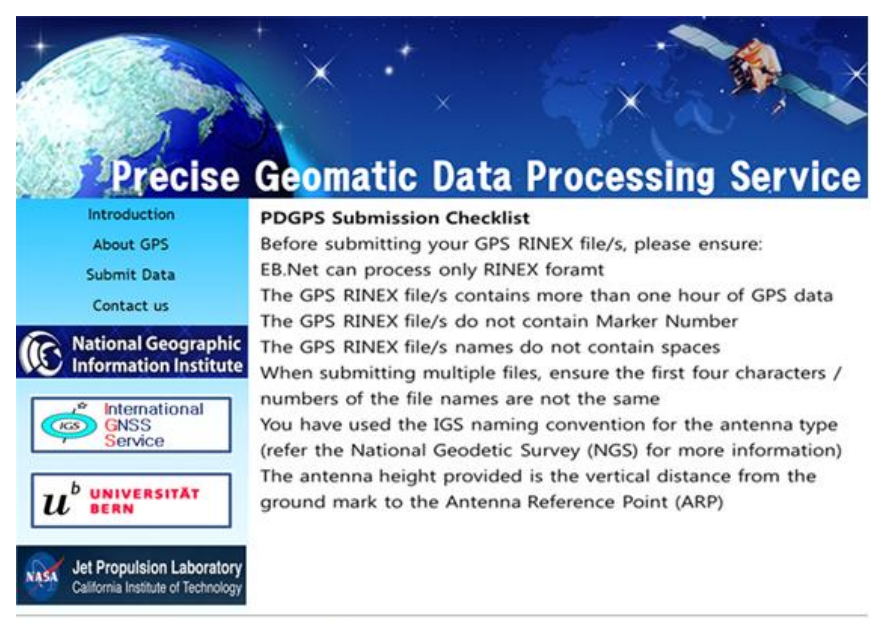

Seoil University

Figure 6. Main screen of the Expert System

Introduction indicates basic development strategies about the solution and system structure. About GPS contains a link to GPS glossary. Submit Data is a menu for GPS data process. Contact us shows developers' emails. Figure 7 displays introduction screen page.

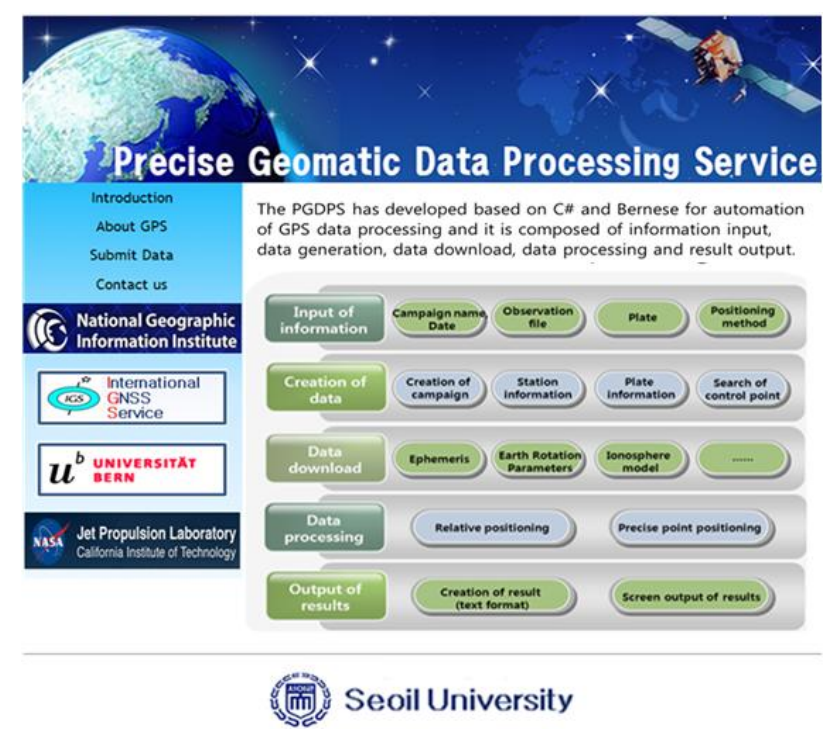

Figure 7. Introduction screen of Expert System

Data Summit is a menu for GPS data process where users' observation data, observation date and email were keyed in. Figures 8 through 10 show Data Summit menu pages for observation data input. 


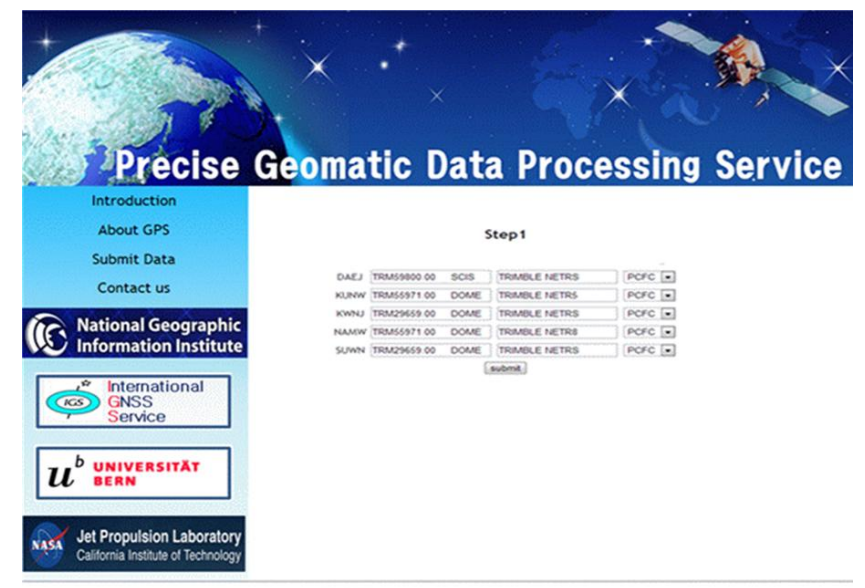

Seoil University

Figure 8. RINEX data input

Input stage of the observation data was based on window for easy and convenient use for users and multiple observation data can be entered. All of the inputted observation data are displayed in page. The system recognizes antenna and receiver types from user input data and automatically displays to screen page. After data input, users key in the observation date and email address to receive the processed results. Figure 9 shows observation date and email input phase.



Figure 9. Date and email input

Observation date is to be keyed in in the form of year, month, day then GPS week and day are automatically calculated for data processing. Result receiving email address is entered in the final stage. Figure 10 is the final confirmation stage of all input information. 


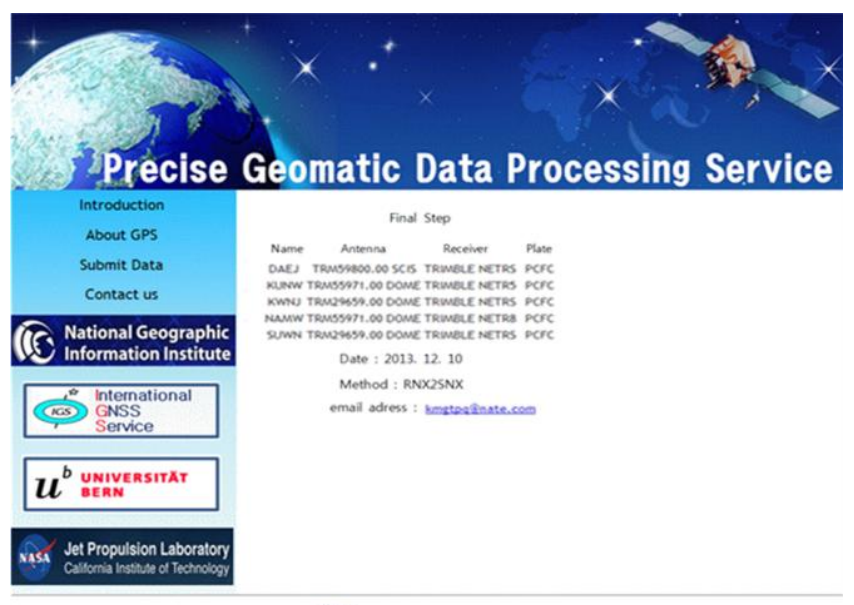

Seoil University

Figure 10. Final step

\section{Comparison of Precise Positioning Process}

In this research, we compared the process of data processing using existing software with the developed expert system to evaluate the applicability. For GPS data-based precise positioning, broad basic knowledge and information about data processing methods and software use are required. To produce precise outcomes, one will need knowledge about softwares, download the related information data such as ephemeris, earth rotation parameters, ionospheric model, and satellite clock anomalies, etc. and convert files to enter in software. Table 2 shows the positioning process using the existing methods and the expert system.

Table 2. Positioning process

\begin{tabular}{ccc}
\hline Process & General Software & Expert System \\
\hline Create campaign & Campaign name & Date \& GPS data \\
\hline Input & Date \& GPS data & \\
Data download & Ephemeris & \\
& $\begin{array}{c}\text { Earth rotation parameters } \\
\text { Satellite clock anomalies } \\
\text { Ionospheric model } \\
\text { Tropospheric model } \\
\text { GPS code bias ... }\end{array}$ & \\
\hline Data creation and & Creation of station file \\
conversion & Conversion of downloaded data \\
& for S/Wning & \\
\hline Data processing & Create satellite orbit & \\
\hline Result & Parameter estimation & \\
\hline
\end{tabular}


Figure 11 compares existing methods with the expert system for positioning process. In the figure, red dotted line parts can be omitted in the expert system. The expert system enables accurate positioning only with the minimum amount of data input, hugely increasing user convenience.

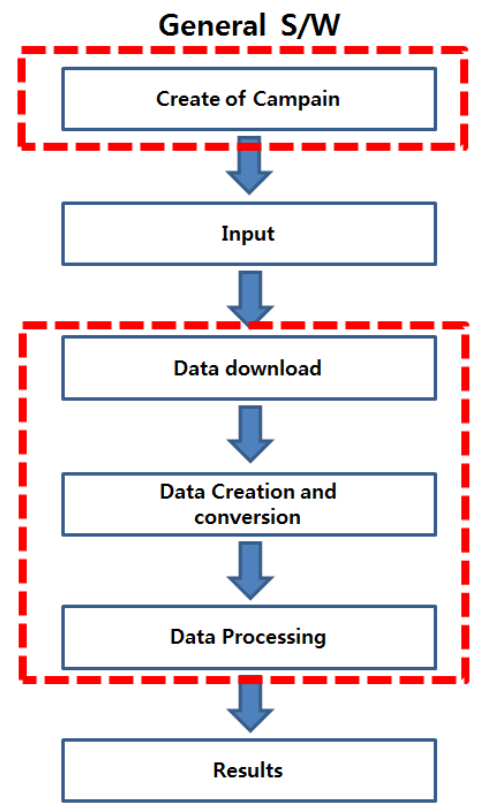

Expert System

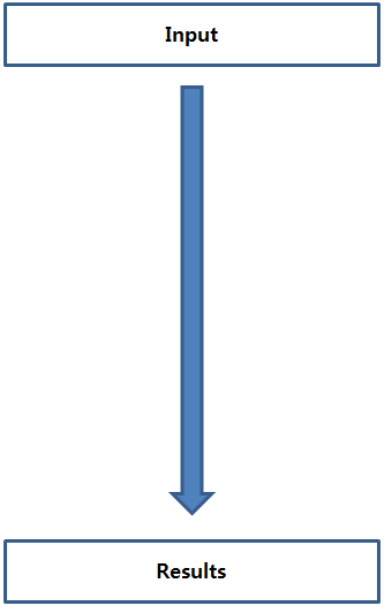

Figure 11. Comparison of positioning process

In general, positioning using GPS, as in Figure 11, requires the creation of campaign, data input, data download, data creation and conversion, data processing and results production. However, the expert system needs only GPS observation data to conduct download, data creation and conversion and data processing automatically and the results are emailed to users, largely saving steps of the process. And as nonprofessionals also can use the system easily, expectations on the system are high for its possibility of versatile application. Figure 12 shows the results of positioning calculated by the expert system. The processing results are written by rectangular plane coordinate (X, Y, Z) in ITRF2000 and longitude, latitude, ellipsoidal height (N, E, H) in GRS80 and it also offers RMSE about the each component [11].

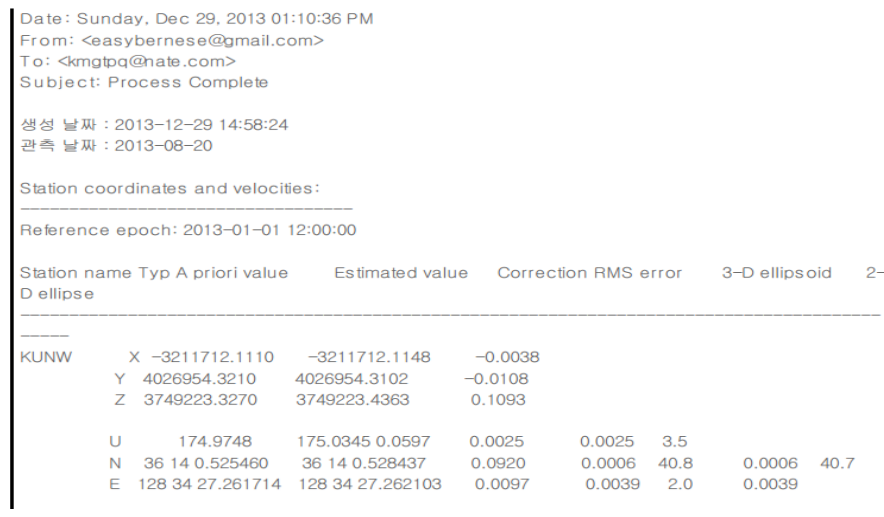

Figure 12. Data processing result using the Expert System 


\section{Conclusion}

This research developed an expert system capable of precise positioning based on GPS observation data even by nonprofessionals. The research outcomes are as follows:

The developed expert system herein adopted an precise science technology software for its inference engine and ephemeris, satellite clock anomalies, ionospheric model, earth rotation parameters, and other parameters needed for positioning as its knowledge base. The user interface applied the MCV model and formed a window for users to easily input their observation data. Nonprofessionals without knowledge or experience in GPS data processing can use the expert system to easily produce reasonable outcomes based on the web. Also, though comparison with other general GPS data processing methods, this research suggested a huge improvement in the model developed herein. The expert system is expected to be much actively applied to the diverse areas with the need to positioning.

\section{Acknowledgements}

This research was supported by Basic Science Research Program through the National Research Foundation of Korea(NRF) funded by the Ministry of Science, ICT \& Future Planning(NRF2012R1A1A1004414).

\section{References}

[1] K. Chen, "Real-Time Precise Point Positioning and Its Potential Applications", Proceedings of the 17th International Technical Meeting of the Satellite Division of The Institute of Navigation (ION GNSS 2004), Long Beach CA, USA, (2004) September 21-24, pp. 1844-1854.

[2] B. W. Parkinson, "Introduction and heritage of NAVSTAR the Global Positioning System", In Parkinson B. W. and J. J. Spilker Jr. Global Positioning System: Theory and Applications Cambridge: American Institute of Aeronautics and Astronautics, vol. 1, (1996), pp. 793.

[3] A. M. Hasan, K. Samsudin and A. R. Ramli, "GPS/INS Integration Based on Dynamic ANFIS Network", International Journal of Control and Automation, vol. 5, no. 3, (2012), pp.1-22.

[4] M. A. Qadeer, A. Chandra and S. Jain, "Design and Implementation of Location Awareness and Sharing System using GPS and 3G/GPRS", International Journal of Multimedia and Ubiquitous Engineering, vol. 7, no. 4 , (2012), pp. 125-140.

[5] J. K. Park, J. S. Lee and M. G. Kim, "Construction of Console Application for Automated GPS Data Processing", International Journal of Control and Automation, vol. 6, no. 1, (2013), pp. 247-254.

[6] J. M. Kang, Y. W. Lee, M. G. Kim and J. K. Park, "Positional Accuracy Analysis of Permanent GPS Sites Using Precise Point Positioning", Journal of the Korean Society of Surveying, Geodesy, Photogrammetry and Cartography, vol. 26, no. 5, (2008), pp. 529-536.

[7] J. W. Kim, J. H. Kwon and J. S. Lee, "The Analysis of the GPS Data Processing of the NGII CORS by Bernese and TGO", Journal of the Korean Society of Surveying, Geodesy, Photogrammetry and Cartography, vol. 26, no. 6, (2008), pp. 549-559.

[8] R. Ghoddousi-Fard and P. Dare, "Online GPS processing services: an initial study", GPS Solut. vol. 10, (2006), pp. 12-20

[9] D. Rolf, H. Urs, F. Pierre and M. Michael, "Bernese GPS Software Version 5.0", Astronomical Institute, University of Bern, (2007), pp. 1-9.

[10] http://en.wikipedia.org.

[11] http://itrf.ensg.ign.fr. 


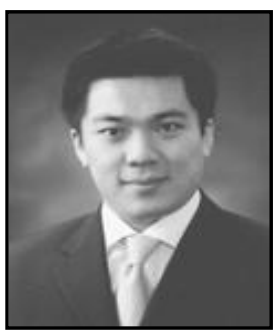

Kap-Yong Jung is currently a post-doctoral researcher in Department of Civil Engineering at Chungnam National University. He received his B.S. degree in Civil Education Engineering, M.S. and Ph.D. degree in Civil Engineering from Chungnam National University in 2003, 2009, 2013, respectively. His research interests are in the areas of GPS, GeoSpatial Information Engineering.

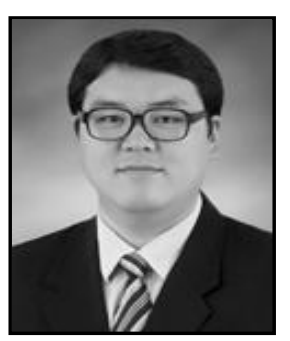

Joon-Kyu Park is currently a Professor in Department of Civil Engineering at Seoil University. He received his B.S., M.S. and Ph.D. degree in Civil Engineering from Chungnam National University in 2001, 2003, 2008, respectively. His research interests are in the areas of GPS, Geo-Spatial Information Engineering. 